\title{
COVID-19 ENHANCED DIMINISHING SENSITIVITY IN PROSPECT-THEORY RISK PREFERENCES: A PANEL ANALYSIS
}

\author{
Shinsuke Ikeda \\ Eiji Yamamura \\ Yoshiro Tsutsui
}

October 2020

The Institute of Social and Economic Research

Osaka University

6-1 Mihogaoka, Ibaraki, Osaka 567-0047, Japan 


\title{
COVID-19 Enhanced Diminishing Sensitivity in Prospect-Theory Risk Preferences: A Panel Analysis*
}

\author{
Shinsuke Ikeda ${ }^{\dagger}$, Eiji Yamamura ${ }^{\ddagger}$, and Yoshiro Tsutsui ${ }^{\S}$
}

October 04, 2020

\begin{abstract}
Based on unique panel data from a five-wave internet survey in Japan, we show how the coronavirus disease 2019 pandemic affected people's prospect-theory risk preferences, especially in the loss domain. The panel analysis indicates that with the spread of the pandemic, diminishing sensitivity becomes stronger for the participants' value and probability weighting functions. Thus, due to the pandemic, (i) people become less sensitive to an increase in losses and feel less pain due to losses, especially large ones; and (ii) they become more pessimistic towards tail loss risks, and more optimistic towards non-tail loss risks. One implication is that people have become less cautious of the risks of suffering large non-tail losses, which might retard the recovery of society.
\end{abstract}

Keywords: COVID-19, prospect theory, risk, value function, probability weighting function. JEL classification: D90, G40.

\footnotetext{
* This research is financially supported by Grants-in-Aid for Scientific Research Fund for the Promotion of Joint International Research (No.18KK0048, Fostering Joint International Research [B]) and for Basic Research (No.17H02499 [B], 15H05728[S], and 20H05631[S]), and Osaka University for its International Joint Research Promotion Program.

$\dagger$ Kwansei Gakuin University and Osaka University (ISER, Guest Professor), E-mail:

ikeda@kwansei.ac.jp

\$ Seinan Gakuin University, E-mail: yamaei@seinan-gu.ac.jp

§ Kyoto Bunkyo University, E-mail: tsutsui@econ.osaka-u.ac.jp 


\section{Introduction}

It is critical to know the effects of pandemics and other external negative shocks on people's risk attitudes. This is because personal risk preferences determine one's preventive and precautionary behaviors against further infestation and the reoccurrence of shocks, respectively. In the framework of the prospect theory (Kahneman and Tsversky, 1978; Tversky and Kahneman, 1992), this study aims to show how the coronavirus disease 2019 (COVID-19, hereafter) affected people's risk attitudes, especially in the loss domain. We use unique panel data from a five-wave internet survey ( $N=14470$ for the balanced panel), conducted from March 2020, when the number of infected individuals started to rise sharply, to June 2020, when the infection rate remained low and the state of emergency was deregulated.

Many studies have reported that risk attitudes are affected by exogenous negative shocks including pandemics (e.g., Bu et al., 2020) and natural disasters (e.g., Page et al., 2014; Cameron and Shah, 2015; Casser et al., 2017; Hanaoka et al. 2018). However, there are two concerns in the literature. First, previous studies were based on the expected utility theory. As is well known (e.g., Wakker, 2010), this theory only has limited capability in describing actual risk attitudes. Second, risk attitudes were examined by eliciting the degree of risk aversion from data on risky choices over positive payoffs, that is, choices in the positive domain. We need to examine risk attitudes in the loss domain because preventive and precautionary behaviors are intrinsic choices between alternative losses. An example of this would be taking a probabilistic risk of suffering an infectious disease versus paying a certain cost of preventive behavior (e.g., wearing a mask, receiving vaccination) and/or precautionary behavior (e.g., getting insurance and saving money).

Motivated by these points, we contribute to the literature by quantifying people's risk attitudes in the loss domain according to the prospect theory. The prospect theory describes people's risky choices by means of (i) the value function, which evaluates outcomes in the gain and loss domains differently; and (ii) the probability weighting function, which describes the subjective impacts of cumulative probabilities (mathematically, probability ranks) and shapes decision weights on outcomes, defined as differences in the probability weight value of cumulative probability.

Experimental data, including those in the seminal articles Kahneman and Tversky (1979) and Tversky and Kahneman (1992), reveal that the value function is concave in the gain domain and convex in the loss domain, while the probability weighting function exhibits an inverse S-shaped curve with respect to objective probability, where people put over-weights on outcomes with extreme cumulative probabilities, that is, near endpoints 0 and 1 of the probability axis, and underweights on those at the middle region of the probability axis (Abdellaoui et al., 2007; Booij et al., 2010; Dhami, 2017). Irrespective of the distinct shapes of the two functions, the resulting risk preferences can be commonly characterized as diminishing sensitivity. The value function reflects that decision makers exhibit diminishing sensitivity to losses and gains; a larger loss (or gain) 
leads to less sensitivity to a marginal increase in loss (or gain). The probability weighting function reflects that the less extreme the underlying cumulative probability is, the less sensitive the decision maker becomes to a change in the probability.

To investigate participants' risk attitude, our survey presented two hypothetical questions for each of the five waves. Participants were asked to choose the highest acceptable insurance premium from a given multiple price list to cover the probabilistic risk of losing a certain amount of money. From the response data, we elicit two risk-preference parameters in prospect theory. The first is the degree of risk tolerance $(1-\alpha)$, where $\alpha$ represents the power parameter of the value function in the loss domain. The second is the degree of distorted impacts of probability $(1-\delta)$, where $\delta$ denotes a power parameter for the probability weighting function in the loss domain. Our interest lies in detecting how the elicited values of these prospect-theory risk parameters change across waves and hence, with the spread of the pandemic.

Our panel analysis shows that as the COVID-19 infection spreads, diminishing sensitivity becomes monotonically stronger for both the value and probability functions; the participants' degree of risk tolerance $(1-\alpha)$, and of probability perception distortion $(1-\delta)$ both continued to rise. On one hand, this implies that people became less sensitive to an increase in losses during the pandemic and therefore, came to feel less pain because of them, particularly large ones. On the other hand, they became more pessimistic about tail loss risks, that is, negative outcomes occurring with small probabilities, and more optimistic to non-tail losses occurring with moderate or large probabilities. Thus, people are considered to have become relatively less cautious than before, especially with the risk of suffering large non-tail losses.

Diminishing sensitivity, measured by $(1-\alpha)$ and $(1-\delta)$, exhibited the sharpest rise at the third wave, which was conducted shortly after the prime minister declared the state of emergency for the seven large prefectures of Japan (Saitama, Chiba, Tokyo, Kanagawa, Osaka, Hyogo, and Fukuoka, prefectures) on April 7. As the declaration had the largest influence on Japanese society and economy, the observed change in the elicited parameters could reflect the total influence of the pandemic, rather than the pure direct effects of virus infection risk. We also found some gender differences. The female sample indicates a stronger diminishing sensitivity than the male sample for both the value and probability weighting functions. Moreover, female participants displayed a faster increase in diminishing sensitivity across waves.

Our finding on the continuous rise of the degree of risk tolerance $(1-\alpha)$ with the spread of the pandemic appears to be inconsistent with the empirical results in the literature that negative shocks, either disasters or stressful events, enhance people's risk aversion (Page et al., 2014; Kandasamy et al., 2014; Cameron and Shah, 2015; Casser, et al., 2017; Bu et al., 2020). In particular, by conducting a two-wave panel survey in Wuhan, China, ground zero of the COVID-19 pandemic, Bu et al. (2020) showed that participants more exposed to the pandemic allocated lower proportions of wealth to risky 
assets in a hypothetical choice question after the outbreak.

This apparent difference could have partially stemmed from the difference in the domain under consideration. Our result considers risk preference in the loss domain, while previous studies considered them in the gain domain. The difference between the two could be reconciled if we interpret the findings in terms of diminishing sensitivity; pandemics and other negative shocks enhance the diminishing sensitivity of the value function either in the gain or loss domain. It is well known that Kahneman and Tversky's (1979) reflection effect, in which risk preferences in the gain domain are the mirror image of those in the loss domain, take place robustly (Fehr-Duda, et. al., 2006; Abdellaoui et al., 2007). To the extent that the reflection property works, our finding that an increase in risk tolerance under COVID-19 could be considered consistent with the literature stating that negative shocks, including the pandemic ( $\mathrm{Bu}$ et al., 2020), enhance risk aversion in the gain domain. Our research is also related to that of $\mathrm{Li}$ et al. (2011) and Page et al. (2014), which showed that natural disasters lead to the display of stronger preferences for prospects with positively skewed prizes, and attributed this to overweighting small probabilities. By detecting the effect on probability weighting, our research provides evidence of their claim.

The remainder of this paper is structured as follows. In Section 2, we explain our data and empirical strategy to elicit prospect-theory risk preference. The results are provided in Section 3. In Section 4, we discuss the possible mechanisms underlying our findings and compare the elicited risk preferences under the pandemic with those during the pre-COVID-19 period that are elicited using data from an independently conducted internet survey. Section 5 concludes this study.

\section{Data and empirical strategy}

\subsection{Panel survey and event flows}

To examine the influence of the COVID-19 pandemic, we administered a five-wave internet panel survey to Japanese respondents between the ages 16 and 79 from March 13 to June 15, 2020. Based on our original questionnaire, the survey was conducted through Intage Inc., a Japanese company with experience in conducting nationwide surveys for both academic and business purposes.

As shown in Figure 1, the survey period covers the main part of the first phase of the pandemic in Japan. The first wave started on March 13, when the Act on Special Measures against Pandemic Influenza was passed, and continued until March 16. It had 4,356 participants who were selected by stratified random sampling such that age and sex distribution were as close as possible to that of the Japanese census. The second wave had 3,495 participants and was conducted from March 27, when the cumulative number of the infected sharply rose to 1,387 , to March 30 .

Figure 1. Infection rates in Japan and survey waves. 
Based on the Act on Special Measures against Pandemic Influenza, Japan's prime minister declared a state of emergency on April 7 for seven large prefectures, including Tokyo and Osaka, to control the pandemic. Although the declaration had no legal force in regulating people's activities, the seven prefectures were requested to keep the guideline of activities in the private and public sectors. This had serious impacts on Japan's society and economy. Soon after the declaration of a state of emergency, the third wave was conducted from April 10 to 13 with 4,013 participants. We conducted the fourth wave from May 8 to 11 with 3,996 participants. By that time, the daily infection rate started to decline, while the state of emergency was extended until the end of May. After the state of emergency was deregulated on May 25, the fifth wave was conducted on June 12 to 15 with 3,877 participants, when society started attempting to return to the pre-pandemic situation.

\subsection{Risk attitude}

In the survey, two hypothetical questions, Q1 and Q2, measured participants' risk attitudes in the loss domain. They had to choose the highest acceptable insurance premium from a given multiple price list to cover a probabilistic risk of losing a certain amount of money. In Q1, respondents are assumed to lose an amount of JPY 100,000 (around USD 1,000) with a 50\% probability, whereas they are supposed to lose JPY 5 million (around USD 50,000) with a probability of $0.1 \%$ (for the precise question in the survey, see Appendix 1) in Q2.

Note that Q1 and Q2 are designed such that they differ in the skewness of the probability distribution of negative outcomes. In Q1, the outcome of losing JPY 0 or JPY 100,000 occurs with a fifty-fifty chance, implying that the distribution has zero skewness. In contrast, participants are supposed to lose a huge amount of money (JPY 5 million) with a small probability $(0.1 \%)$ in Q2, in which case, skewness is highly negative (-997). ${ }^{1}$ As the prospect theory shows, people with an inverse S-shaped probability weighting function tend to be averse to probabilistic losses with a negatively skewed distribution; they are pessimistic about rare negative events. ${ }^{2}$ By examining the responses to both questions with different skewness, we can clearly characterize the participants' risk attitudes by means of the value and probability weighting functions.

We suppose that each participant responds to Q1 and Q2 by evaluating the (negative) value of the corresponding probabilistic losses based on their prospect theory values $V_{1}$ and $V_{2}$. We let $x_{i} \quad(i=1,2)$ denote the negative outcome of question Q $i \quad\left(x_{1}=-100,000\right.$; and $x_{2}=$ $-5,000,000)$ and $p_{i}$, the probability that $x_{i}$ occurs $\left(p_{1}=0.5\right.$; and $\left.p_{2}=0.001\right)$. Accordingly, the prospect theory value of the probabilistic loss supposed in question $\mathrm{Q} i$ is evaluated using the value

${ }^{1}$ Skewness of a distribution is defined as the third-order moment of the z-value distribution.

2 In contrast, people with an inverse S-shaped probability weighting function in the gain domain prefer probabilistic gains with a positively-skewed distribution; they are optimistic about rare positive outcomes, such as lotteries. 
function $v$ and the probability weighting function $w$ as $^{3}$ :

$$
V_{i}=w\left(p_{i}\right) v\left(x_{i}\right), \quad i=1,2 .
$$

Following Tversky and Kahneman (1992), we specify functions $v(x)$ and $w(x)$ in the loss domain $(x<0)$ as:

$$
\begin{gathered}
v(x)=-\lambda(-x)^{\alpha}, \\
w(p)=\frac{p^{\delta}}{\left(p^{\delta}+(1-p)^{\delta}\right)^{\frac{1}{\delta}}},
\end{gathered}
$$

where parameter $\alpha(0 \leq \alpha \leq 1)$ for the value function determines the degree of risk tolerance $(1-\alpha)$ in the loss domain, and $\delta(0 \leq \delta \leq 1)$ for the probability weighting function determines the depth $(1-\delta)$ of the inverse S-shaped curve. Parameter $\lambda$ captures the degree of loss aversion. However, as the current study does not consider prospects in which negative and positive outcomes are mixed, the participants' choices do not depend on $\lambda$.

Let $R_{i}(i=1,2)$ be the highest acceptable insurance premium revealed from the response data to question $\mathrm{Q} i$. By construction, the prospect-theory values are equalized between paying $R_{i}$ and taking a risk of negative prospect $\left(x_{i}, p_{i} ; 0,1-p_{i}\right)$ :

$$
v\left(-R_{i}\right)=w\left(p_{i}\right) v\left(x_{i}\right), i=1,2 .
$$

Substituting (2) and (3) into this equation yields the following:

$$
\begin{aligned}
\left(R_{1}\right)^{\alpha} & =\frac{0.5^{\delta}(100000)^{\alpha}}{\left\{0.5^{\delta}+(1-0.5)^{\delta}\right\}^{1 / \delta}}, \\
\left(R_{2}\right)^{\alpha} & =\frac{0.001^{\delta}(5000000)^{\alpha}}{\left\{0.001^{\delta}+(1-0.001)^{\delta}\right\}^{1 / \delta}}
\end{aligned}
$$

where $\lambda$ disappears because it is canceled out. By solving these simultaneous equations, we can obtain the values of $\alpha$ and $\delta$ for each participant. By taking the natural log of the equations and

${ }^{3}$ Precisely, cumulative prospect theory uses the decision weighting function $\pi$ to decide the weight of an outcome $x$ occurring with probability $p$, where $\pi$ is defined as a difference in the value of the probability weighting function defined over cumulative probability (probability rank). However, in the negative prospects considered in Q1 and Q2, there are only two outcomes, zero and a negative outcome, so that from the definition of $\pi$ (Tversky and Kahneman, 1992) we have $\pi\left(p_{i}\right)=w\left(p_{i}\right)$ for $p_{i}$, the probability that the worst outcome (suffering a loss) occurs. 
rearranging the results, we have the following two equations:

$$
\begin{gathered}
\frac{\ln \left(R_{2}\right)-\ln (5000000)}{\ln \left(R_{1}\right)-\ln (100000)}=\frac{\delta \ln (0.001)-\left(\frac{1}{\delta}\right) \ln \left(0.001^{\delta}+(1-0.001)^{\delta}\right)}{\delta \ln (0.5)-\left(\frac{1}{\delta}\right) \ln \left(0.5^{\delta}+(1-0.5)^{\delta}\right)}, \\
\alpha=\frac{\delta \ln (0.5)-\left(\frac{1}{\delta}\right) \ln \left(0.5^{\delta}+(1-0.5)^{\delta}\right)}{\ln \left(R_{1}\right)-\ln (100000)} .
\end{gathered}
$$

For the values of the highest acceptable insurance premiums $R_{1}$ and $R_{2}$ that each participant revealed in Q1 and Q2, the value of parameter $\delta$ in the probability weighting function is obtained from (4) for each respondent. In turn, the value of parameter $\alpha$ for the value function is computed from (5) for each participant given the value of $\delta$.

\section{Results}

\subsection{Mean comparison}

Table 1 summarizes the cross-wave changes in the participants' acceptable insurance premiums $\left(R_{1}, R_{2}\right)$ in questions $\mathrm{Q} 1$ and $\mathrm{Q} 2$, and the risk premiums implied therefrom, $R_{1} / 50,000-1$ for $\mathrm{Q} 1$ and $R_{2} / 5000-1$ for $\mathrm{Q} 2$. Note that a positive risk premium implies risk-averse behavior, whereas a negative one implies a risk-tolerant choice. From Table 1, we can see two distinct tendencies. First, participants are consistently risk tolerant for Q1 and risk averse for Q2 through all five waves. This is consistent with the prospect theory's prediction that people are risk tolerant when evaluating a prospect defined over the loss domain, insofar as the payoff probability distribution is not so much negatively skewed, as in Q1. The same theory states that people are risk averse in the loss domain when the associated distribution is greatly negatively skewed, as in Q2.

Table 1. Summary statistics of insurance premiums revealed from Questions Q1 and Q2.

Second, and more importantly, Table 1 shows that as the wave proceeds and COVID-19 spreads, risk premiums implied from Q1 and Q2 decrease almost monotonically. This implies that participants become more risk tolerant in Q1 and less risk averse in Q2, that is, their behavior becomes more risk tolerant on average as the pandemic spreads.

Our main interest is in how these behavioral tendencies that occur with the spread of COVID-19 are quantified in terms of risk preference parameters in the prospect theory. There are three noteworthy points from Table 2, which shows the summary statistics for the elicited values of prospect theory parameters $\alpha$ and $\delta$ in each wave.. First, the mean values of $\alpha$ and $\delta$ are both between 0 and 1 in 
each wave. This implies that the value function and probability weighting function elicited, on average, have a diminishing sensitivity property that prospect theory predicts. That is, the average participant has a convex value function in the loss domain, displaying diminishing sensitivity to marginal increases in the loss amount. The probability weighting function in the loss domain of average participants is inversely S-shaped, and hence, has the diminishing sensitivity property: overweighting occurs for tail loss risks, whereas underweighting takes place for non-tail loss risks.

Table 2. Summary statistics of prospect theory parameters in the loss domain

Second, the mean values of $\alpha$ and $\delta$ monotonically decrease as the wave proceeds, suggesting that diminishing sensitivity tends to be stronger for both the value and probability weighting functions. The elicited mean cross-wave shifts of both functions are depicted in Figures 2(a) and 2(b). As COVID-19 spreads, the value function becomes more curved, and the inverse $\mathrm{S}$ shape of the probability weighting function becomes deeper.

Figure 2. Cross-wave shifting of the value and the probability weighting functions
(a) The value function
(b) The probability weighting function

Using the balanced panel sample, Figures 3(a) and (b) show the statistical significance of these preference shifts, where comparisons are made for the male, female, and full samples. In particular, for either of the male or female sample, $\alpha$ and $\delta$ both exhibit the sharpest, statistically significant declines in the third wave. As the third wave was conducted shortly after the government declared the state of emergency, the largest reductions in the risk parameters could reflect the direct and indirect impacts of the declaration. Except for significant reductions in the female participants' $\alpha$ in the second wave, other decreases in $\alpha$ and $\delta$ between two consecutive waves are not statistically significant. Note, however, that these results are based on simple mean comparison, without controlling for fixed effects. With fixed effects being controlled, cross-wave differences and, consequently, the influences of the outbreak of COVID-19 become more significant, as we show in the next section.

Figure 3. Cross-wave comparison of prospect theory parameters in the loss domain
(a) The value function
(b) The probability weighting function

We can also find gender differences in Figures 3(a) and (b). Both $\alpha$ and $\delta$ are lower for females than males, implying that the females tend to exhibit a stronger diminishing sensitivity for both prospect-theory functions. This result is consistent with that of Fehr-Duda et al. (2006), who showed 
through laboratory experiments that women's elicited probability weighting function tends to be more curved than men's in either the gain or loss domain. In particular, the study detected the same tendency in the context of insurance evaluation, as in this study. Our findings are consistent with these results. ${ }^{4}$

Our finding that females' degree of risk tolerance in the loss domain is larger than that in males seems to contradict the stylized tendency that the former is more risk averse than the latter (e.g., Croson and Gneezy, 2009). However, previous research did not control for possible gender differences in probability weighting. After controlling for gender difference in probability weighting, Fehr-Duda et al. (2006) found no cross-gender difference in the degree of risk tolerance with respect to the value function. Both their results and ours suggest that gender differences in risk aversion could differ from what the previous literature claimed.

\subsection{Regressions}

To confirm the findings in the previous subsection, we estimate a fixed effect model by regressing prospect-theory parameters on four wave dummy variables for the $2^{\text {nd }}$ to $5^{\text {th }}$ waves, Wave2-Wave5, where Wave 1 is the reference case. In doing so, we control daily COVID-19 infections, Infected COVID19, measured based on the daily numbers of those who tested positive in the polymerase chain reaction (PCR) test in prefectures where participants live.

Table 3 summarizes the estimation results. First, in either the male, female, or full sample, both risk parameters $\alpha$ and $\delta$ decline, and diminishing sensitivity $1-\alpha$ and $1-\delta$ rises across waves. Particularly, as in Figure 3, such impacts occur significantly in Wave 3, that is, just after the declaration of the state of emergency. The coefficients of Wave 3 in absolute value are almost more than triple those of Wave 2. In addition, unlike the simple $t$-tests in Figure 3, the panel analysis shows that marginal reductions in $\alpha$ and $\delta$ between two consecutive waves are all significant, except for those between Waves 3 and 4 . This means that $1-\alpha$ and $1-\delta$ continue to move up, that is, diminishing sensitivity is monotonically enhanced for the value function and the probability weighting function in association with the spread of COVID-19.

Table 3. Fixed effect estimations

Enhanced diminishing sensitivity in two prospect-theory functions has different implications for the influence of the pandemic on risk attitudes. With the value function exhibiting stronger diminishing sensitivity, that is, with $(1-\alpha)$ continuously increasing, people gradually become less sensitive to

\footnotetext{
${ }^{4}$ Figures 3(a) and (b) also show that cross-wave reductions, especially between waves 2 and 3, are larger for females than males.

${ }^{5}$ In Section 4.2, we will show that another data set indicates the same gender difference that females display stronger diminishing sensitivity.
} 
an increase in losses, and consequently feel less pain from given losses. For example, for average participants in Wave 5 , the pain of losing JPY 5 million, measured by $\mid v\left(J P Y ~ 5\right.$ million) $\mid{ }^{6}$ is only 0.33 times that of Wave 1 .

Note that such a reduction in feeling pain $|v(x)|$ against a loss $x(<0)$ due to an increase in $(1-$ $\alpha)$ becomes larger as the loss amount $|x|$ increases. Thus, in association with the spread of COVID19, people feel less pain from large losses (e.g., losing JPY 5 million) at a higher speed than they do with smaller losses (e.g., losing JPY 1000). Therefore, they become less cautious about large losses, relative to smaller ones. ${ }^{7}$

As the probability weighting function enhances its diminishing sensitivity property under the pandemic, the overweighting of tail losses and underweighting of non-tail losses become stronger. For example, the probability weight that average participants put on a negative outcome occurring with probability $0.1 \%$, that is, $w(0.1 \%)$, becomes larger from $4.7 \%$ in Wave 1 to $5.6 \%$ in wave 5 , implies that overweighting becomes stronger. In contrast, the probability weight $w(50 \%)$ decreases from $29.1 \%$ in Wave 1 to $26.3 \%$ in wave $5,{ }^{8}$ that is, underweighting for non-tail losses is enhanced under the spread of the pandemic.

In sum, COVID-19 affects people's risk attitudes in two ways. First, due to the pandemic, people become less sensitive to an increase in losses, particularly when they are large. Second, people become more pessimistic about tail loss risks, and more optimistic about non-tail loss risks. The two findings imply that people are the least sensitive to a large non-tail loss risk. Policymakers should pay attention to this side effect as the risk of large non-tail losses is widely prevalent (e.g., risk of virus infection when staying home with infected family members, risk of developing cancer when smoking, risk of global warming, etc.), which could weaken people's preventive and/or precautionary behavior against large risks.

These results relate to the existing literature in the following ways. First, the result that the degree of risk tolerance in the loss domain continuously rose with the spread of the pandemic appears to differ from the empirical results in the literature which stated that negative shocks, either disasters or stressful events, enhance risk aversion (Page et al., 2014; Kandasamy et al., 2014; Cameron and Shah, 2015; Casser, et al., 2017; Bu et al., 2020). However, these studies, except Kandasamy et al. (2014), did not control for the effect on probability weighting. More importantly, previous studies measured risk aversion in the gain domain, while the present study considers the loss domain. Although the results

\footnotetext{
${ }^{6}$ Average participants' pain of losing JPY 5 million, measured by $\mid v$ (JPY 5 million)|, amounts to 1203.13 for Wave 1, 897.79 for Wave 2, 480.91 for Wave 3, 466.20 for Wave 4, and 399.66 for Wave 5.

7 Pain of losing JPY 1,000 at Wave 5 becomes 0.61 times as much as that in Wave 1, compared to 0.33 for pain of JPY 5 million.

8 The value of $w(0.1 \%)$ is $4.7 \%$ for Wave $1,4.9 \%$ for Wave $2,5.4 \%$ for waves 3 and 5 , and $5.6 \%$ for Wave 5. The value of $w(50 \%)$ amounts to $29.1 \%$ for Wave $1,28.4 \%$ for Wave $2,26.9 \%$ for Wave $3,26.7 \%$ for Wave 4 , and $26.3 \%$ for Wave 5 .
} 
may be different with respect to the effects on the degree of risk aversion, it could be reconciled if we interpret the results in terms of diminishing sensitivity. The findings of previous studies and those of ours commonly indicate that pandemics and other negative shocks strengthen diminishing sensitivity to a marginal increase in losses and gains. The reflection effect, in which risk preferences in the gain domain are the mirror image of those in the loss domain, is known to be robust (Kahneman and Tversky, 1979; Fehr-Duda et al., 2006; Abdellaoui et al., 2007; Dhami, 2016). This interpretation is considered to have certain validity.

Second, Li et al. (2011) and Page et al. (2014) showed that natural disasters lead people to display stronger preferences for prospects with positively skewed prizes. They attributed this to the increased overweighting of small probabilities. However, they did not estimate the probability weights or control for the effect on the value function. Our finding that people's probability weighting exhibits stronger diminishing sensitivity under the pandemic provides evidence to their claim.

\section{Discussions}

\subsection{Possible mechanisms}

We could consider two mechanisms in which the spread of the COVID-19 pandemic affected participants' risk attitude: mental stress and liquidity shortage.

\subsubsection{Mental stress}

The spread of the COVID-19 infection directly or indirectly had serious negative effects on mental health. For example, Yamamoto et al. (2020) reported that 33.6\% of 11,333 participants experienced mild-to-moderate psychological distress, and $11.5 \%$ suffered from serious mental distress. Mental stress is known to affect risk attitude (Haushofer \& Fehr, 2014; Cohn et al., 2015). Therefore, mental stress brought about by the COVID-19 pandemic could affect people's risk attitudes. Our results could be interpreted as reflecting the influence of mental stress caused by COVID-19.

In this sense, our results are comparable with the findings of Kandasamy et al. (2014), who gave participants a dose of hydrocortisone and examined how experimentally raised stress hormone (cortisol) levels affected their risk attitudes. Their findings were captured by the shapes of the value and probability weighting functions. The authors showed that experimentally raised cortisol levels (i) made the participants' value function in the gain domain more concave, wherein they became more risk averse in the gain region; and (ii) deepened the inverse $\mathrm{S}$ shape of the weighting probability function for male participants, that is, overweighting of positive tail outcomes and underweighting of positive non-tail outcomes are more exaggerated under increased stress for males. If our result reflects the stress effect of the pandemic, our finding on its effect on the probability weighting function is consistent with, and even stronger, than that of Kandasamy et al. (2014) as stated in (ii), in which the 
same effect was not detected in the female sample. ${ }^{9}$ Moreover, our finding relates to Kandasamy et al.'s (2014) result (i); both commonly indicate that stressful events enhance the diminishing sensitivity of the value function.

\subsubsection{Liquidity shortage}

As COVID-19 had a persistent negative income effect, ${ }^{10}$ another plausible mechanism could be the lack of liquidity; under liquidity shortage, participants might become more reluctant to buy insurance as negative income shocks continue (McDermott et al., 2014), which could lead to reductions in acceptable risk premiums in Q1 and Q2. To examine the validity of this hypothesis, we re-estimate the fixed effect model by adding to the set of independent variables the product terms of wave dummies and a low income dummy, which equal one when the participant belongs to the bottom $10 \%$ income class, and zero otherwise. If the lack-of-liquidity hypothesis holds, the coefficients of the product terms are negative.

Table 4. Fixed effects model with interaction terms with a low income-group indicator

As seen in Table 4, the hypothesis is invalid. Contrary to the prediction of the hypothesis, the coefficients of the interaction terms of wave dummies and the low income group indicator are all positive and highly significant, especially for the $\alpha$ estimation. This result could indicate the rational risk-insuring behavior of low-income participants. Relative to those in higher income groups, they became more willing to buy insurance in Q1 and Q2 because of poor self-insurance ability under the pandemic.

\subsection{Comparing to pre-COVID-19 risk preference}

We have interpreted that the observed changes in the $\alpha$ and $\delta$ values observed during the pandemic period reflect some level of influence, direct or indirect, of the pandemic. However, the finding would be weak because the elicited parameter values are based on the data after the outbreak of the pandemic and not compared with those in the pre-pandemic period

To address this limitation, we can use comparable data from the NTT Human Information Data 2018 (NTTHID2018, hereafter), a large-scale internet survey conducted in Japan by NTT Human Information Research Institute, Inc. in October 2018 (N=20,160). Participants were randomly selected from adults in Japan. In the survey, one of the authors in charge of designing the questionnaire, asked participants questions similar to Q1 and Q2, where hypothetical loss amounts were JPY 10,000, rather

\footnotetext{
${ }^{9}$ Moreover, in Kandasamy et al. (2014), the elicited probability weighting function has an irregular S shape, not an inverse $\mathrm{S}$ shape, for the placebo male sample and treatment female sample.

${ }^{10}$ For example, seasonally-adjusted quarterly gross domestic product (GDP) on the expenditure base dropped by $5.93 \%$ from the $4^{\text {th }}$ quarter of FY 2019 to the $1^{\text {st }}$ quarter of FY 2020 (Monthly GDP ReportJune, 2020, Japan Center for Economic Research).
} 
than JPY 100,000 in Q1, and JPY 50,000 rather than JPY 5 million. The probability setting is the same as in Q1 and Q2. As participants were randomly selected widely from adults in Japan, as in this panel survey, a comparison of the elicited risk attitude between the NTTHID2018 sample and that of the present panel data could provide certain information on how people's risk attitudes differ between the pre- and post-outbreak periods of the pandemic. If our conclusion that the pandemic enhances diminishing sensitivity for prospect-theory risk preference is supported, diminishing sensitivity in the pre-COVID-19 period, elicited from the NTTHID2018 sample, will be weaker than it is in the present panel data. This result will be especially applicable in the Wave 1 sample, for which diminishing sensitivity is the weakest among the five wave samples.

Table 5. Comparison to pre-COVID-19 risk preference

Table 5 compares the mean values for $\alpha$ and $\delta$ between the Wave 1 sample of the present panel data and the pre-COVID-19 sample of NTTHID2018. For both male and female samples, the preCOVID-19 mean values of $\alpha$ and $\delta$ are significantly larger than the corresponding post-COVID-19 values, that is, the Wave 1 mean values. This could be taken as indirect evidence that the observed cross-wave increase in diminishing sensitivity for prospect-theory risk preferences reflect the influence of the COVID-19 pandemic.

In addition, the table shows that also in the NTTHID2018 sample, females' $\alpha$ and $\delta$ are on average smaller than males', implying that they exhibit stronger diminishing sensitivity than males in the loss domain, consistent with the gender difference found in Figure 3.

\section{Conclusions}

Based on the analysis of a unique five-wave panel survey in Japan, we found that diminishing sensitivity in the loss domain became stronger for people's prospect-theory risk preference with the spread of the COVID-19 pandemic. Thus, (i) losses, particularly large losses, became less painful; and (ii) people became more pessimistic toward tail loss risks, but more optimistic toward nontail loss risks.

This research provides new insights in understanding the effect of pandemics and other negative shocks on risk attitudes. First, the effects are captured in terms of changes on those on diminishing sensitivity, rather than on risk aversion/tolerance. Our findings and those of the literature's commonly indicate that pandemics and other negative shocks enhance diminishing sensitivity in risk evaluation. Second, by detecting the distorting effect of the pandemic on probability weighting, we provide strong empirical evidence to the previous finding that disasters lead those affected to avoid tail loss risks ( $\mathrm{Li}$ et al., 2011; Page, 2014). Our finding on this point could also be taken as evidence to Bu et al.'s (2020) conjecture that changes in people's risk attitudes after the outbreak could occur because of their 
pessimistic beliefs on various probabilistic environments.

Findings (i) and (ii) imply that, after pandemic outbreaks and other negative shocks, people become much less cautious to the risk of non-tail, large losses. Because the risk of large non-tail losses is widely prevalent (e.g., the risk of virus infection when joining a meeting/party, risk of developing cancer when smoking, risk of global warming, etc.), policymakers should take this side effect into careful consideration, as it could weaken people's preventive and/or precautionary behavior against large risks.

Further research is necessary to strengthen our study. First, to the best of our knowledge, there is no explanation on how neural activity relates to the S-shaped curvature of the value function that underlies the reflection effect property (Fox and Poldrack, 2009), which we rely on to reconcile our result to that of the literature. The effect on risk attitude should be compared in detail between the positive and negative domains. Second, we need to examine how our results relate to the effect of mental stress. In particular, with the important exception of Kandasamy et al. (2014), there have been few attempts to examine how mental stress affects prospect theory preferences captured by the value function and the probability weighting function. Third, it is important to examine how the effects of negative shocks, including pandemics and natural disasters, are interacted with people's loss-aversion inclination. Such shocks may affect people's degree of loss aversion, which will in turn change their preventive and precautionary behaviors. This issue has been left unexamined. 


\section{References}

Abdellaoui, M., H. Bleichrodt, and C. Paraschiv, 2007, Loss aversion under prospect theory: A parameter-free measurement, Management Science 53, 1659-1674.

Booij, A.S., B.M.S. van Praag, and G. van de Kuilen, 2010, A parametric analysis of prospect theory's functionals for the general population, Theory Decision 68, 115-148.

Borghans, L., B.H.H. Golsteyn, J.J. Heckman, and H. Meijers, 2009, Gender differences in risk aversion and ambiguity aversion, Journal of European Economic Association 7, 649-658.

Bu, D., T. Hanspal, Y. Lao and Y. Liu, 2020, Risk taking during a global crisis: Evidence from Wuhan, Covid Economics 5, 106-146.

Byrnes, J.P., D.C. Miller, and W.D. Schafer, 1999, Gender differences in risk taking: A meta-analysis, Psychological Bulletin 125, 367-383.

Cahlı'kova', J. and L. Cingl, 2017, Risk preferences under acute stress, Experimental Economics 20, 209-236.

Cameron, L. and M. Shah, Risk-taking behavior in the wake of natural disasters, Journal of Human Resources 50, 484-515.

Cassar, A., A. Healy, and C. von Kessler, 2017, Trust, risk, and time preference after a natural disaster: Experimental evidence from Thailand, World Development 94, 90-105.

Charness, G. and U. Gneezy, 2012, Strong evidence for gender differences in risk taking, Journal of Economic Behavior \& Organization 83, 50-58.

Cohn, A., J. Engelmann, E. Fehr, and M. A. Maréchal, 2015, Evidence for countercyclical risk aversion: An experiment with financial professionals, American Economic Review 105, 860885 .

Croson, R. and U. Gneezy, 2009, Gender differences in preferences, Journal of Economic Literature $47,448-474$.

Dhami, S. (2016), The Foundation of Behavioral Economic Analysis, Oxford, UK: Oxford U.P.

Fehr-Duda, H., M. De Gennaro, and R. Schubert, 2006, Gender, financial risk, and probability weights, Theory and Decision 60, 283-313.

Fox, C.R. and R.A. Poldrack, 2009, Prospect theory and the brain, in: Glimcher, P.W., C.F. Camerer, E. Fehr, and R.A. Poldrack eds., Neuroeconomics Decision Making and the Brain, London, UK: Elsevier.

Hanaoka, C., H. Shigeoka, and Y. Watanabe, 2018, Do risk preferences change? Evidence from the Great East Japan Earthquake, American Economic Journal: Applied Economics 10, 298-330.

Haushofer, J. and E. Fehr, 2014, On the psychology of poverty, Science 344, 862-867.

Kahneman, D. and A. Tversky, 1979, Prospect theory: An analysis of decision under risk, Econometrica 47, 263-291. 
Kandasamy, N., B. Hardy, L. Page, M. Schaffner, J. Graggaber, A.S. Powlson, P.C. Fletcher, M. Gurnell, and J. Coates, 2014, Cortisol shifts financial risk preferences, Proceedings of National Academy of Science 111, 3608-3613.

Li, J., S. Li, W. Wang, L. Rao, and H. Liu, 2011, Are people always more risk averse after disasters? Surveys after a heavy snow-hit and a major earthquake in China in 2008, Applied Cognitive Psychology 25, 104-111.

McDermott, T.K.J., F. Barry, and R.S.J. Tol, 2014, Disasters and development: natural disasters, credit constraints, and economic growth, Oxford Economic Papers 66, 750-773.

Page, L. D.A. Savage, and B. Torgler, 2014, Variation in risk seeking behavior following large losses: A natural experiment, European Economic Review 71, 121-131.

Tversky, A. and D. Kahneman, 1992, Advances in prospect theory: Cumulative representation of uncertainty, Journal of Risk and Uncertainty 5, 297-323.

Wakker, P.P., 2010, Prospect Theory: For Risk and Uncertainty, Cambridge: Cambridge U.P. 
Appendix Questions Q1 and Q2.

Q1. Assume that there is a 50\% risk of losing JPY 100,000 on a given day. You can take out insurance to cover this amount in case of a loss. What is the maximum amount you would pay to purchase the insurance? (Place an $\mathrm{X}$ in ONE box.)

1. Not purchase even if the price is JPY 0 .

$\square$ 2. Purchase if the price is less than or equal to JPY 1,000 .

$\square$ 3. Purchase if the price is less than or equal to JPY 5,000.

4. Purchase if the price is less than or equal to JPY 10,000.

$\square$. Purchase if the price is less than or equal to JPY 15,000 .

$\square$ 6. Purchase if the price is less than or equal to JPY 20,000.

7 . Purchase if the price is less than or equal to JPY 30,000.

$\square$ 8. Purchase if the price is less than or equal to JPY 40,000 .

9. Purchase if the price is less than or equal to JPY 45,000 .

10. Purchase if the price is less than or equal to JPY 50,000 .

11 . Purchase even if the price is more than JPY 50,000.

Q2. Assume that there is a $0.1 \%$ risk of losing JPY 5 million on a given day. You can take out insurance to cover this amount in case of a loss. What is the maximum amount you would pay to purchase the insurance? (Place an $\mathrm{X}$ in ONE box.)

1. Not purchase even if the price is JPY 0.

$\square$ 2. Purchase if the price is less than or equal to JPY 1,000 .

$\square$ 3. Purchase if the price is less than or equal to JPY 5,000.

$\square$ 4. Purchase if the price is less than or equal to JPY 10,000 .

$\square$ 5. Purchase if the price is less than or equal to JPY 20,000 .

$\square$ 6. Purchase if the price is less than or equal to JPY 30,000 .

$\square$ 7. Purchase if the price is less than or equal to JPY 50,000.

$\square$ 8. Purchase if the price is less than or equal to JPY 100,000.

$\square$ 9. Purchase if the price is less than or equal to JPY 500,000 .

$\square$ 10. Purchase if the price is less than or equal to JPY 1 million.

$\square$ 11. Purchase even if the price is more than JPY 1 million. 
Table 1 Summary statistics of insurance premiums revealed from Questions Q1 and Q2

\begin{tabular}{|c|c|c|c|c|c|c|c|c|c|c|}
\hline \multirow{5}{*}{$\begin{array}{l}\text { Question } \\
\text { Insurenace covering: } \\
\text { Skewness }\end{array}$} & \multicolumn{5}{|c|}{ Q1 } & \multicolumn{5}{|c|}{$\mathbf{Q 2}$} \\
\hline & \multirow{2}{*}{\multicolumn{5}{|c|}{$\begin{array}{c}(-\mathrm{JPY} 100,000,0.5) \\
\text { zero }\end{array}$}} & \multicolumn{5}{|c|}{ (-JPY5,000,000, 0.001) } \\
\hline & & & & & & & highly & negative $(-C$ & & \\
\hline & \multicolumn{2}{|c|}{$\begin{array}{l}\text { Acceptable premium } \\
\qquad\left(\mathrm{JPY} R_{1}\right)\end{array}$} & \multicolumn{2}{|c|}{$\begin{array}{l}\text { Implied risk premium } \\
\qquad\left(R_{1} / 50000-1\right)\end{array}$} & \multirow[b]{2}{*}{ Obs. } & \multicolumn{2}{|c|}{$\begin{array}{l}\text { Acceptable premium } \\
\qquad\left(\mathrm{JPY} R_{2}\right)\end{array}$} & \multicolumn{2}{|c|}{$\begin{array}{l}\text { Implied risk premium } \\
\qquad\left(R_{2} / 5000-1\right)\end{array}$} & \multirow[b]{2}{*}{ Obs. } \\
\hline & Mean & S.D. & Mean & S.D. & & Mean & S.D. & Mean & S.D. & \\
\hline Wave 1 & 8420.939 & 12012.320 & -0.832 & 0.240 & 4359 & 24357.270 & 116271.800 & 3.871 & 23.254 & 4359 \\
\hline Wave 2 & 7586.738 & 11094.220 & -0.848 & 0.222 & 3495 & 18878.390 & 98852.760 & 2.776 & 19.771 & 3495 \\
\hline Wave 3 & 5586.488 & 9726.284 & -0.888 & 0.195 & 4013 & 11746.140 & 70110.110 & 1.349 & 14.022 & 4013 \\
\hline Wave 4 & 5340.297 & 9384.288 & -0.893 & 0.188 & 3996 & 11769.930 & 71504.250 & 1.354 & 14.301 & 3996 \\
\hline Wave 5 & 4924.136 & 9033.211 & -0.902 & 0.181 & 3877 & 10087.990 & 66166.880 & 1.018 & 13.233 & 3877 \\
\hline all & 6386.616 & 10423.990 & -0.872 & 0.208 & 19740 & 15472.860 & 87400.350 & 2.095 & 17.480 & 19740 \\
\hline
\end{tabular}

Note: This summarizes basic statistics of participants' acceptable insurance premiums that are revealed by their responses to questions Q1 and Q2 at each of five waves. Implied risk premiums represent the acceptable rates of risk premium that are implied from acceptable insurance premiums. A positive (negative) implied risk premium implies risk averse (tolerant) behavior. 
Table 2 Summary statistics of prospect-theory parameters

\begin{tabular}{lrrrrr}
\hline & \multicolumn{3}{c}{$\alpha$} & \multicolumn{2}{c}{$\delta$} \\
\cline { 2 - 5 } & Mean & \multicolumn{1}{c}{ S.D. } & Mean & \multicolumn{1}{c}{ S.D. } & Obs. \\
Wave 1 & 0.464 & 0.234 & 0.425 & 0.131 & 4321 \\
Wave 2 & 0.443 & 0.210 & 0.419 & 0.115 & 3470 \\
Wave 3 & 0.403 & 0.176 & 0.401 & 0.110 & 3991 \\
Wave 4 & 0.399 & 0.177 & 0.399 & 0.110 & 3983 \\
Wave 5 & 0.389 & 0.170 & 0.395 & 0.104 & 3860 \\
all & 0.420 & 0.198 & 0.408 & 0.115 & 19625 \\
\hline
\end{tabular}

Note: Parameter $\alpha$ represents the power of the value function in the loss domain (see equation (2)), where the degree of risk tolerance is given by $(1-\alpha)$, so that a higher $\alpha$ implies a lower degree of risk tolerance. Parameter $\delta$ is one for the probability weighting function in the loss domain (see equation (3)). A smaller $\delta$ value implies that the inverse $\mathrm{S}$ shape of the probability weighting function is deeper. 
Table 3. Fixed effect model estimations.

\begin{tabular}{|c|c|c|c|c|c|c|}
\hline & \multicolumn{3}{|c|}{ Dep. Variable $=\boldsymbol{\alpha}$} & \multicolumn{3}{|c|}{ Dep. Variable $=\boldsymbol{\delta}$} \\
\hline & Full & Male & Female & Full & Male & Female \\
\hline Wavel & & $<$ default $>$ & & & $<$ default $>$ & \\
\hline Wave2 & $\begin{array}{l}-0.019^{* * *} \\
(0.004)\end{array}$ & $\begin{array}{l}-0.014^{* *} \\
(0.007)\end{array}$ & $\begin{array}{l}-0.025^{* * *} \\
(0.005)\end{array}$ & $\begin{array}{l}-0.007^{* * *} \\
(0.003)\end{array}$ & $\begin{array}{l}-0.008^{* *} \\
(0.003)\end{array}$ & $\begin{array}{l}-0.006^{*} \\
(0.003)\end{array}$ \\
\hline Wave3 & $\begin{array}{l}-0.059^{* * *} \\
(0.004)\end{array}$ & $\begin{array}{l}-0.053^{* * *} \\
(0.006)\end{array}$ & $\begin{array}{l}-0.065^{* * *} \\
(0.005)\end{array}$ & $\begin{array}{l}-0.025^{* * *} \\
(0.003)\end{array}$ & $\begin{array}{l}-0.021^{* * * *} \\
(0.004)\end{array}$ & $\begin{array}{l}-0.029 * * * \\
(0.003)\end{array}$ \\
\hline Wave4 & $\begin{array}{l}-0.058^{* * *} \\
(0.004)\end{array}$ & $\begin{array}{l}-0.049^{* * *} \\
(0.007)\end{array}$ & $\begin{array}{l}-0.068^{* * *} \\
(0.006)\end{array}$ & $\begin{array}{l}-0.025^{* * *} \\
(0.003)\end{array}$ & $\begin{array}{l}-0.024^{* * *} \\
(0.003)\end{array}$ & $\begin{array}{l}-0.028^{* * *} \\
(0.004)\end{array}$ \\
\hline Wave5 & $\begin{array}{l}-0.068^{* * *} \\
(0.005)\end{array}$ & $\begin{array}{l}-0.061^{* * *} \\
(0.007)\end{array}$ & $\begin{array}{l}-0.074^{* * *} \\
(0.006)\end{array}$ & $\begin{array}{l}-0.030^{* * *} \\
(0.003)\end{array}$ & $\begin{array}{l}-0.029^{* * * *} \\
(0.004)\end{array}$ & $\begin{array}{l}-0.032^{* * *} \\
(0.003)\end{array}$ \\
\hline Waves 2 vs 3 & $\mathrm{P}=0.00$ & $\mathrm{P}=0.00$ & $\mathrm{P}=0.00$ & $\mathrm{P}=0.00$ & $\mathrm{P}=0.00$ & $\mathrm{P}=0.00$ \\
\hline Waves 3 vs 4 & $\mathrm{P}=0.79$ & $\mathrm{P}=0.45$ & $\mathrm{P}=0.45$ & $\mathrm{P}=0.60$ & $\mathrm{P}=0.35$ & $\mathrm{P}=0.70$ \\
\hline Waves 4 vs 5 & $\mathrm{P}=0.00$ & $\mathrm{P}=0.00$ & $\mathrm{P}=0.07$ & $\mathrm{P}=0.00$ & $\mathrm{P}=0.04$ & $\mathrm{P}=0.07$ \\
\hline $\begin{array}{l}\text { Within R-Sq. } \\
\text { Groups } \\
\text { Obs. }\end{array}$ & $\begin{array}{l}0.05 \\
2,894 \\
14,470\end{array}$ & $\begin{array}{l}0.03 \\
1,458 \\
7,290\end{array}$ & $\begin{array}{l}0.08 \\
1,436 \\
7,180\end{array}$ & $\begin{array}{l}0.03 \\
2,894 \\
14,470\end{array}$ & $\begin{array}{l}0.02 \\
1,458 \\
7,290\end{array}$ & $\begin{array}{l}0.04 \\
1,436 \\
7,180\end{array}$ \\
\hline
\end{tabular}

Note: Parameters $\alpha$ and $\delta$ represent ones for the value function and the probability weighting function, respectively, in the loss domain (see equations (2) and (3)). The number of persons infected with COVID 19 is included as a control variable. However, it does not show statistical significance and its results are not reported. Numbers within parentheses are robust standard errors clustered on individuals. ${ }^{* *}$ and $* *$ indicate the statistical significance at $1 \%$ and $5 \%$ levels, respectively. 
Table 4. Fixed effect model with interaction terms with a low income group indicator

\begin{tabular}{|c|c|c|c|c|c|c|}
\hline & \multicolumn{3}{|c|}{ Dep. Variable $=\boldsymbol{\alpha}$} & \multicolumn{3}{|c|}{ Dep. Variable $=\boldsymbol{\delta}$} \\
\hline & Full & Male & Female & Full & Male & Female \\
\hline $\begin{array}{l}\text { Wave } 2 \times \\
\text { Bottom 10\% }\end{array}$ & $\begin{array}{l}0.021^{*} \\
(0.011)\end{array}$ & $\begin{array}{l}0.029 \\
(0.019)\end{array}$ & $\begin{array}{l}0.013 \\
(0.012)\end{array}$ & $\begin{array}{l}0.003 \\
(0.006)\end{array}$ & $\begin{array}{l}0.001 \\
(0.001)\end{array}$ & $\begin{array}{l}0.005 \\
(0.009)\end{array}$ \\
\hline $\begin{array}{l}\text { Wave } 3 \times \\
\text { Bottom 10\% }\end{array}$ & $\begin{array}{l}0.027 * * * \\
(0.008)\end{array}$ & $\begin{array}{l}0.015 \\
(0.013)\end{array}$ & $\begin{array}{l}0.038 * * * \\
(0.010)\end{array}$ & $\begin{array}{l}0.005 \\
(0.006)\end{array}$ & $\begin{array}{l}-0.005 \\
(0.010)\end{array}$ & $\begin{array}{l}0.016 * * \\
(0.008)\end{array}$ \\
\hline $\begin{array}{l}\text { Wave } 4 \times \\
\text { Bottom 10\% }\end{array}$ & $\begin{array}{l}0.024 * * * \\
(0.008)\end{array}$ & $\begin{array}{l}0.019 \\
(0.013)\end{array}$ & $\begin{array}{l}0.028 * * \\
(0.011)\end{array}$ & $\begin{array}{l}0.012 * \\
(0.006)\end{array}$ & $\begin{array}{l}0.012 \\
(0.009)\end{array}$ & $\begin{array}{l}0.011 \\
(0.008)\end{array}$ \\
\hline $\begin{array}{l}\text { Wave } 5 \times \\
\text { Bottom } 10 \%\end{array}$ & $\begin{array}{l}0.031 * * * \\
(0.009)\end{array}$ & $\begin{array}{l}0.029 * * \\
(0.013)\end{array}$ & $\begin{array}{l}0.032 * * * \\
(0.012)\end{array}$ & $\begin{array}{l}0.019 * * * \\
(0.007)\end{array}$ & $\begin{array}{l}0.021 * * \\
(0.010)\end{array}$ & $\begin{array}{l}0.018^{* *} \\
(0.009)\end{array}$ \\
\hline Wavel & & $<$ default $>$ & & & $<$ default $>$ & \\
\hline Wave2 & $\begin{array}{l}-0.022 * * * \\
(0.004)\end{array}$ & $\begin{array}{l}-0.017^{* *} \\
(0.007)\end{array}$ & $\begin{array}{l}-0.027 * * * \\
(0.006)\end{array}$ & $\begin{array}{l}-0.008 * * * \\
(0.003)\end{array}$ & $\begin{array}{l}-0.008^{* *} \\
(0.004)\end{array}$ & $\begin{array}{l}-0.007 * \\
(0.004)\end{array}$ \\
\hline Wave3 & $\begin{array}{l}-0.062 * * * \\
(0.004)\end{array}$ & $\begin{array}{l}-0.054 * * * \\
(0.007)\end{array}$ & $\begin{array}{l}-0.069^{* * *} \\
(0.005)\end{array}$ & $\begin{array}{l}-0.025 * * * \\
(0.003)\end{array}$ & $\begin{array}{l}-0.020^{* * *} \\
(0.004)\end{array}$ & $\begin{array}{l}-0.030 * * * \\
(0.003)\end{array}$ \\
\hline Wave4 & $\begin{array}{l}-0.061 * * * \\
(0.005)\end{array}$ & $\begin{array}{l}-0.051^{* * *} \\
(0.007)\end{array}$ & $\begin{array}{l}-0.071 * * * \\
(0.006)\end{array}$ & $\begin{array}{l}-0.027 * * * \\
(0.003)\end{array}$ & $\begin{array}{l}-0.025^{* * *} \\
(0.004)\end{array}$ & $\begin{array}{l}-0.029 * * * \\
(0.004)\end{array}$ \\
\hline Wave 5 & $\begin{array}{l}-0.071 * * * \\
(0.005)\end{array}$ & $\begin{array}{l}-0.065^{* * *} \\
(0.008)\end{array}$ & $\begin{array}{l}-0.078^{* * *} \\
(0.006)\end{array}$ & $\begin{array}{l}-0.033 * * * \\
(0.003)\end{array}$ & $\begin{array}{l}-0.032 * * * \\
(0.004)\end{array}$ & $\begin{array}{l}-0.034 * * * \\
(0.004)\end{array}$ \\
\hline $\begin{array}{l}\text { Within R-Sq. } \\
\text { Groups } \\
\text { Obs. }\end{array}$ & $\begin{array}{l}0.05 \\
2,894 \\
14,470 \\
\end{array}$ & $\begin{array}{l}0.04 \\
1,458 \\
7,290\end{array}$ & $\begin{array}{l}0.08 \\
1,436 \\
7,180\end{array}$ & $\begin{array}{l}0.03 \\
2,894 \\
14,470 \\
\end{array}$ & $\begin{array}{l}0.02 \\
1,458 \\
7,290\end{array}$ & $\begin{array}{l}0.04 \\
1,436 \\
7,180\end{array}$ \\
\hline
\end{tabular}

Note: Bottom 10\% represents a binary indicator which equals one when the participant's household belongs to the bottom $10 \%$ income group. The number of persons infected with COVID-19 is included as a control variable although it's result is not reported. Numbers within parentheses are robust standard errors clustered on individuals. $* * *$ and $* *$ indicate the statistical significance at $1 \%$ and $5 \%$ levels, respectively. 
Table 5. Comparison to the pre-COVID19 risk preference

\begin{tabular}{|c|c|c|c|c|}
\hline & \multicolumn{4}{|c|}{0} \\
\hline & \multicolumn{2}{|c|}{ Male } & \multicolumn{2}{|c|}{ Female } \\
\hline & \multicolumn{2}{|r|}{ NTTHID2018 } & \multicolumn{2}{|r|}{ NTTHID2018 } \\
\hline Mean & 0.471 & 0.618 & 0.457 & 0.566 \\
\hline (S.D.) & $(0.251)$ & $(0.283)$ & $(0.216)$ & $(0.262)$ \\
\hline$t$-value & \multicolumn{2}{|c|}{24.129} & \multicolumn{2}{|c|}{20.525} \\
\hline \multirow[t]{4}{*}{ obs. } & 2185 & 10365 & 2136 & 9591 \\
\hline & \multicolumn{4}{|c|}{$\delta$} \\
\hline & \multicolumn{2}{|c|}{ Male } & \multicolumn{2}{|c|}{ Female } \\
\hline & Wave 1 & NTTHID2018 & Wave 1 & NTTHID2018 \\
\hline Mean & 0.4317 & 0.501 & 0.418 & 0.447 \\
\hline (S.D.) & $(0.144)$ & $(0.204)$ & $(0.116)$ & $(0.170)$ \\
\hline$t$-value & \multicolumn{2}{|c|}{18.600} & \multicolumn{2}{|c|}{9.182} \\
\hline obs. & 2185 & 10365 & 2136 & 9591 \\
\hline
\end{tabular}

Note: The table compares the mean values of the prospect-theory parameters $\alpha$ and $\delta$ in the loss domain in the wave 1 sample of the present panel data and those in the NTTHID2018 data. NTTHID2018 is a web survey conducted in October, 2018 independently of this research, where similar questions in the loss domain to Q1 and 
Figure 1. COVID-19 infection rates in Japan and survey waves.

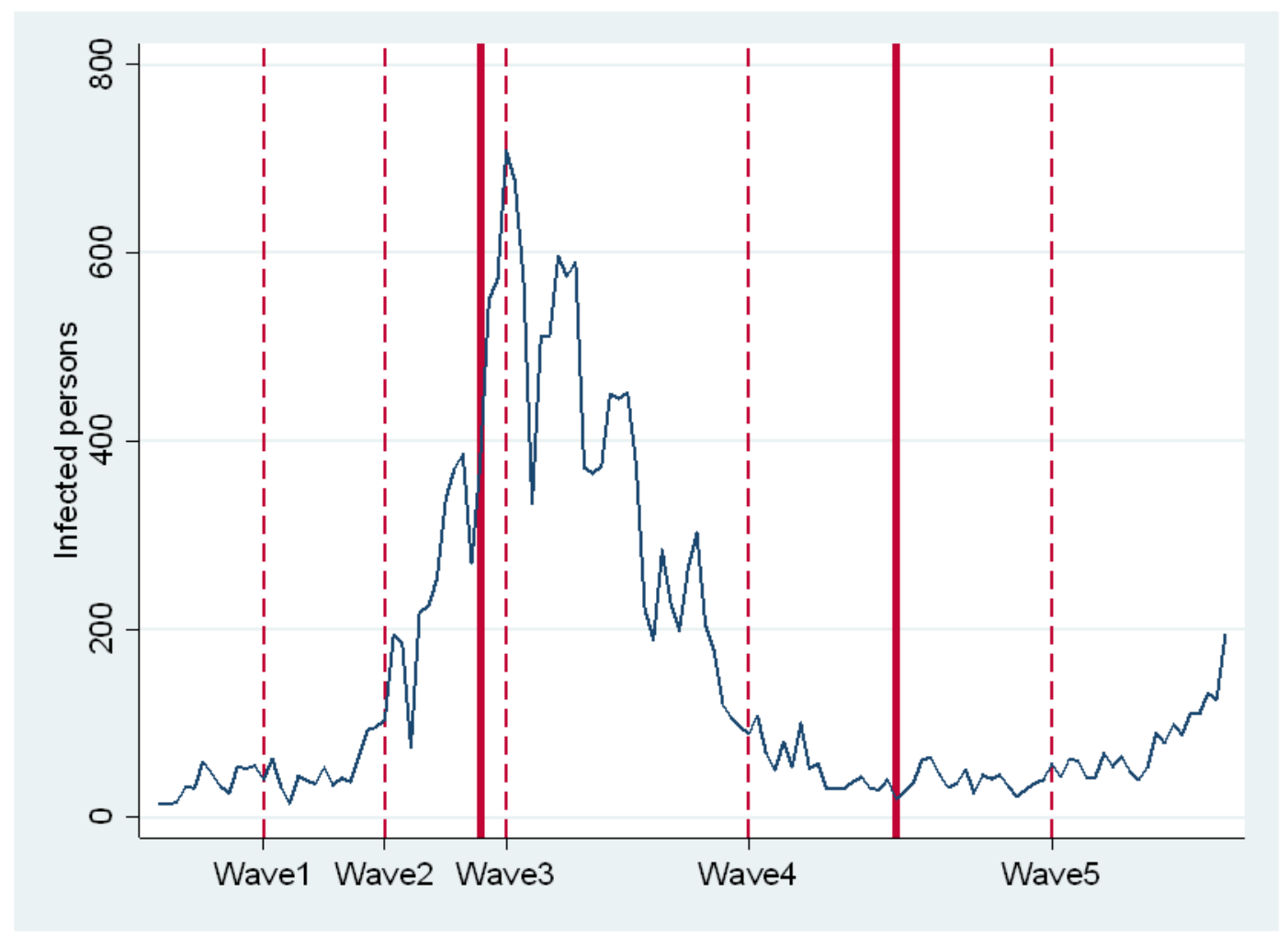

Note: The graph depicts cross-wave development of the daily numbers of persons getting infected COVID-19 in Japan, measured by the daily numbers of persons who are in the positive PCR test. The first, second, third, fourth and fifth waves were conducted on Mach 13-16, March 27-30, April 10-13, May 8-11, and June 12-15, respectively. Dashed lines indicate the date of the surveys. Two bold lines show the dates when the state of emergency was declared (April 7) and when the state of emergency was deregulated (May 25). 
Figure 2. Cross-wave shifting of the value and the probability weighting functions in the loss domain.

(a) The value function

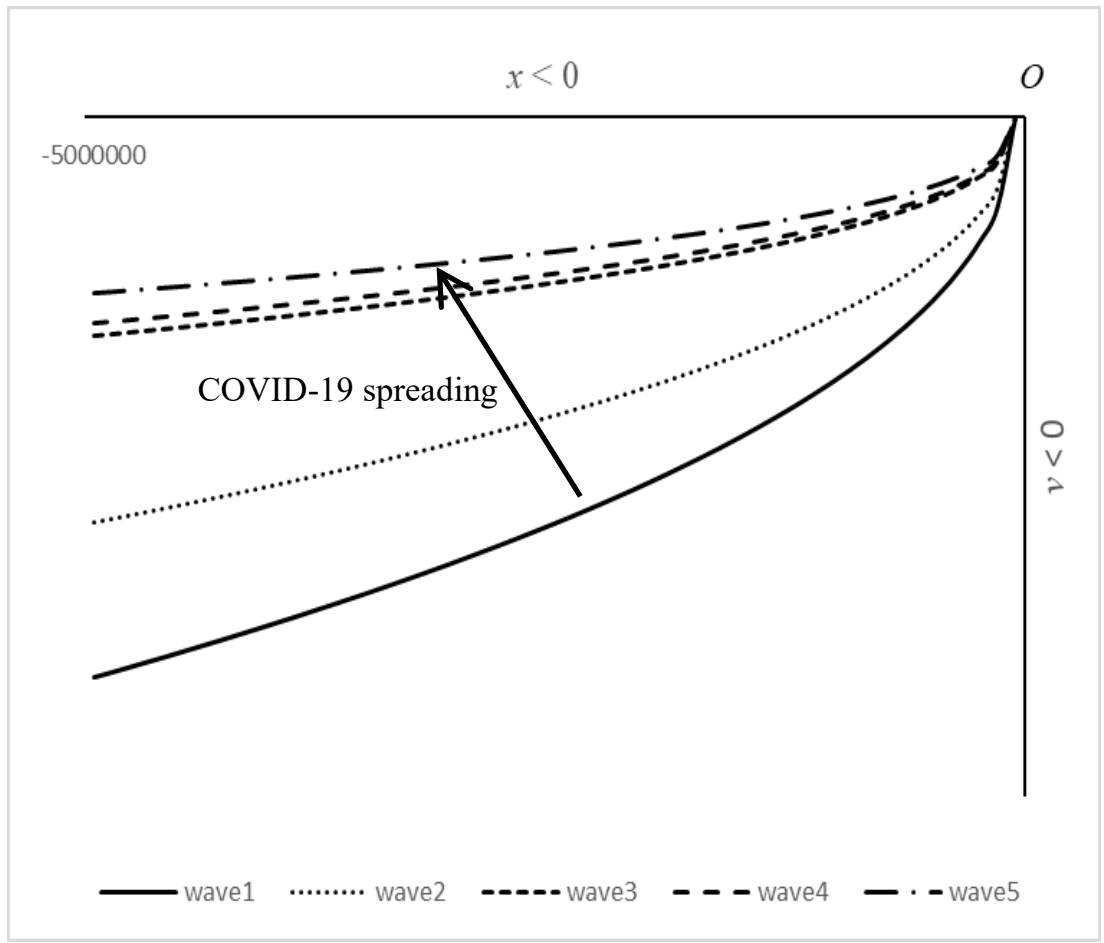

(b) The probability weighting function

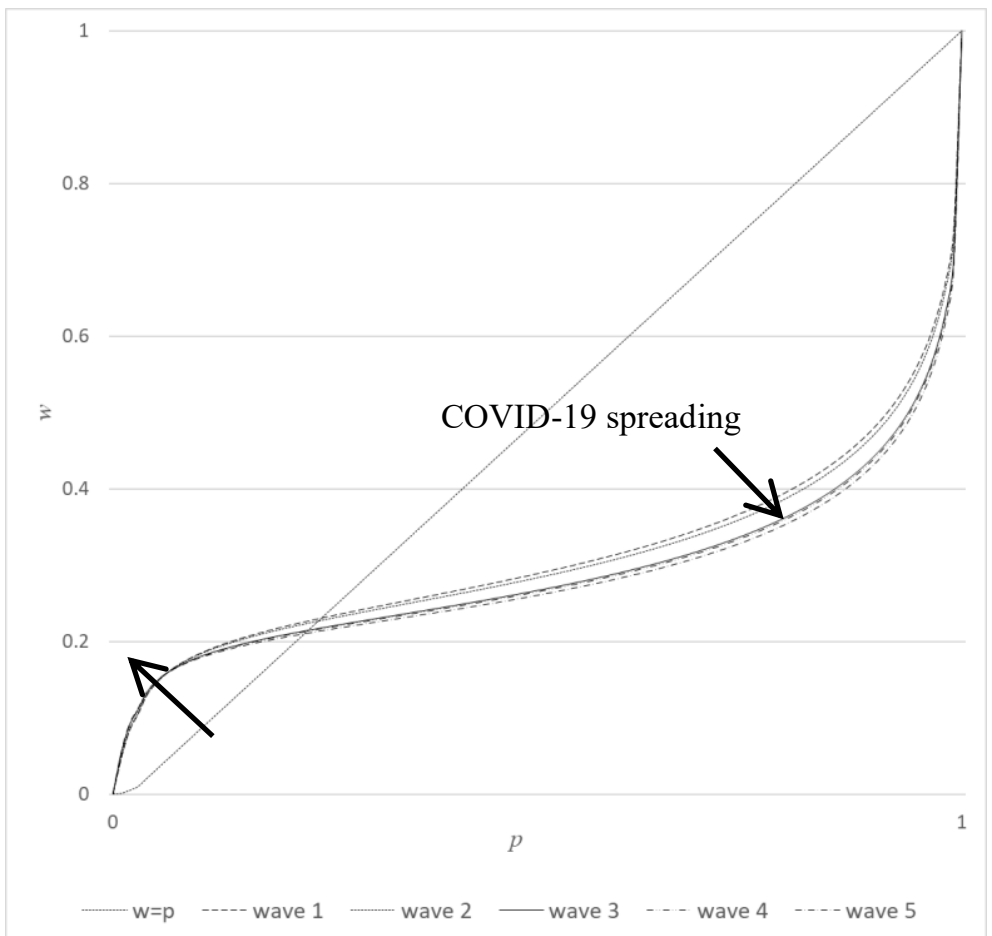

Note: The figures depict cross-wave shifting of the value function ((a)) and the probability weighting function ((b)), where both are defined over the loss domain. The loci of the functions are computed using mean values of $\alpha$ and $\delta$ at each wave summarized in Table 2. For the value function, we set $\lambda=$ 2.25, the median value obtained by Tversky and Kahneman (1993). 
Figure 3. Cross-wave comparison of prospect theory parameters in the loss domain (a) $\alpha$

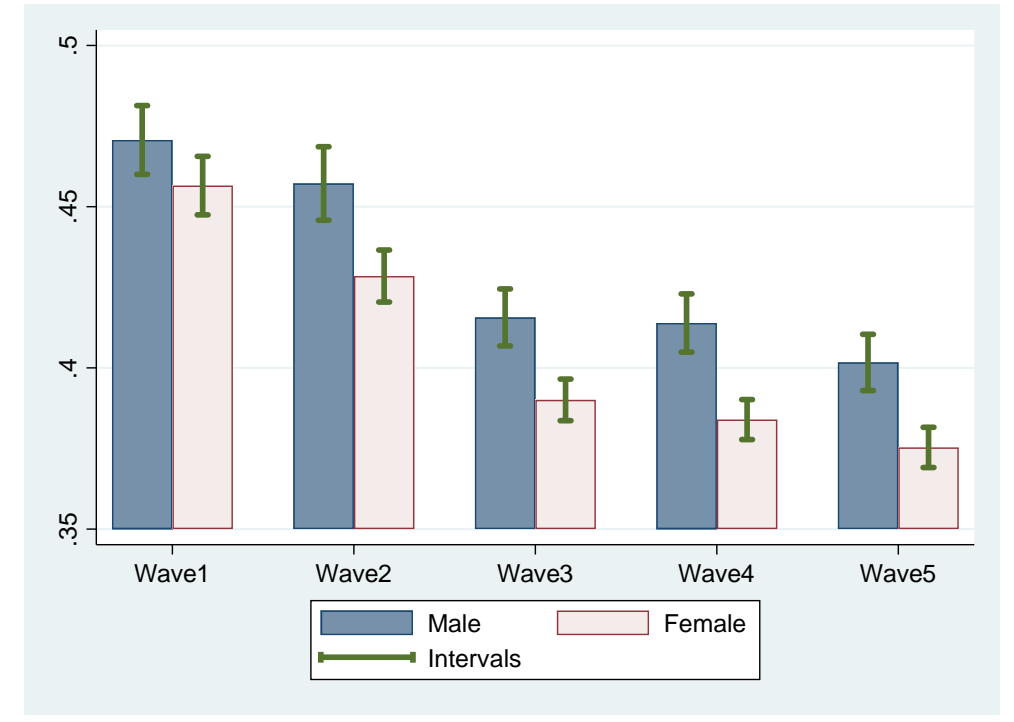

(b) $\delta$

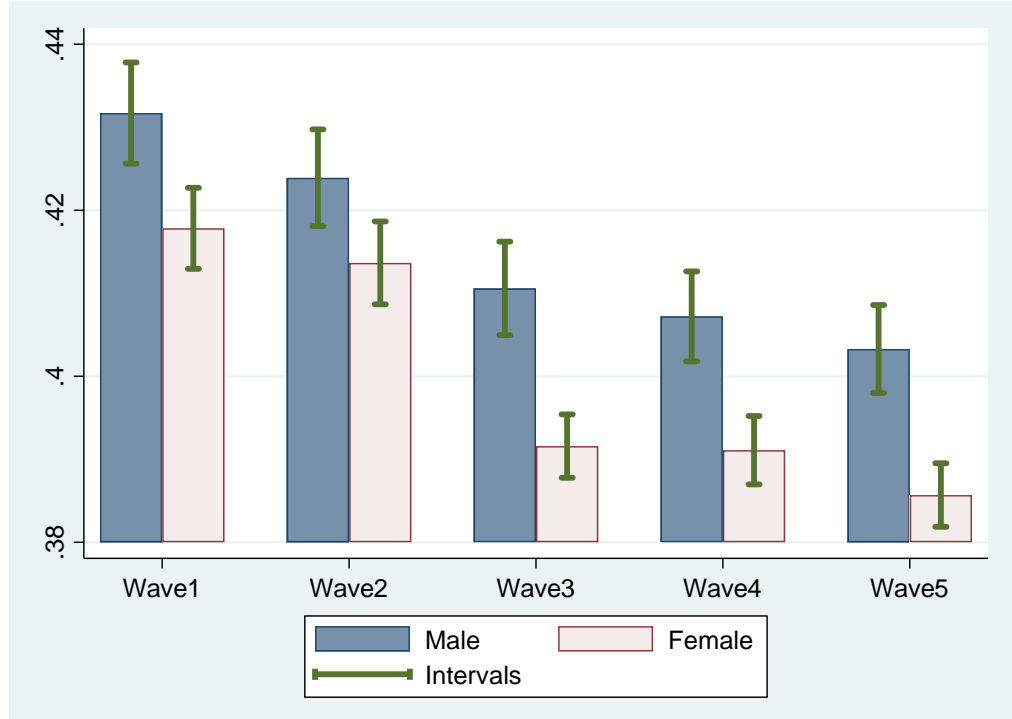

Note: The balanced panel data are used $(N=14470)$. Parameters $\alpha$ and $\delta$ are those of the value and probability weighting functions, respectively. The first, second, third, fourth and fifth waves were conducted on Mach 13-16, March 27-30, April 10-13, May 8-11, and June 12-15, respectively. The intervals represent the $95 \%$ confidence intervals. 